\title{
Virtual and mixed reality intelligent environments
}

\author{
Michael Gardner ${ }^{\mathrm{a}}$, Jonathon Richter ${ }^{\mathrm{b},{ }^{*}}$ and Aki Härmä ${ }^{\mathrm{c}}$ \\ ${ }^{a}$ University of Essex, School of Computer Science and Electronic Engineering, Wivenhoe Park, Colchester, \\ CO43SQ, UK \\ ${ }^{\mathrm{b}}$ University of Oregon, Center for Learning in Virtual Environments, P.O. Box 8013, Missoula, MT 59807, USA \\ ${ }^{\mathrm{c}}$ Philips Research, High Tech Campus 36, 5656AE, Eindhoven, The Netherlands
}

\section{Background}

This thematic issue addresses core topics on Virtual and Mixed Reality Intelligent Environments. The conventional definitions for virtual and mixed reality systems, e.g., in [1-3], do not require that such environments should be intelligent or even reactive. However, most useful applications of virtual reality can be considered intelligent in the sense that the environment changes and reacts to the actions of the user. This journal has traditionally focused on ambient intelligence and smart environments which are considered to be real physical environments with sensors and actuators rather than artificial intelligent environments based on software only. The goal of this thematic issue is to bring these two concepts together.

It is clear that applications based on physical sensors and actuators, and virtual reality technologies will coexist in future environments. In addition, one may assume that the intelligence and the way that a system can react intelligently, should be similar in real, mixed, and virtual environments. In mixed reality, intelligent behavior could be through the adaptation of the elements of the virtual environment to the objects of the physical space, or the adaptation of the objects of the physical space to the elements of the virtual or mixed reality, and the adaptation of the physical or virtual environment to the preferences or behaviors of the users.

\footnotetext{
"Corresponding author. E-mail: jrichter@uoregon.edu.
}

\section{About this thematic issue}

New application concepts based on intelligent environments are often very challenging to test and evaluate because building such environments and getting all the necessary technologies working together may be difficult and time consuming. Therefore it is often a good idea to use a virtual reality system as a laboratory for testing new applications. However, this requires that the simulation of the real environment and the users in the virtual environment is sufficiently realistic. The holy grail is an artificial intelligence system which controls the behavior of avatars in a virtual environment as if they were real people and in this way allow testing of complex intelligent environments without actually building them. The paper by Marc Davies proposes an interesting intermediate solution where the behavior of avatars in a virtual reality simulation is controlled based on data collected from users in a real environment.

The idea of virtual reality is often associated with large projection screens in CAVE installations or head-mounted display systems. Therefore, it is interesting to note that smart phones are now becoming one of the most important end-user platforms for virtual and mixed reality applications and technologies. Smart phones are present in our daily life and new applications which allow for the adaptation of the content on the screen (the 'virtual reality') to the real physical environment around the user may lead to beneficial services for a user. The paper by Olsson et al. evaluates several examples of mobile augmented reality application concepts in a series of user 
tests. The experiments show that the concepts can make contextually relevant information easily available and allow novel interaction possibilities with the physical world. However, the study also gives several important user insights relevant to the design and evaluation of such systems.

The paper by Laffey et al. provides a framework for smart collaborative environments based on environment scaffolding, social affordances and coaching, which are illustrated in the iSocial virtual world learning environment. This provides valuable insights for designers wishing to build smart environments that can adapt to the needs of the users, and it provides a heuristic for thinking about smart 3D environments as a complete ecosystem.

\section{References}

[1] H. Tamura, H. Yamamoto and A. Katayama, Mixed reality: Future dreams seen at the border between real and virtual worlds, IEEE Computer Graphics and Appl. 21 (2001 Nov./ Dec.), 64-70.

[2] P. Milgram and F. Kishino, A taxonomy of mixed reality visual display, IEICE Trans. Inform. and Sys. E77-D(12) (1994), 1321-1329.

[3] M.W. Krueger, Artificial Reality II, Addison-Wesley, Reading, MA, 1991. 